http://jmscr.igmpublication.org/home/ ISSN (e)-2347-176x ISSN (p) 2455-0450 crossref DOI: https://dx.doi.org/10.18535/jmscr/v8i4.71

\title{
Assessment of Quality of Life and Medication Adherence in Hypertensive Patients in Tertiary Care Hospital
}

Authors

\author{
Dr K. Mahitha Reddy ${ }^{*}$, Dr M. Apoorva Varma, Dr C.Nithish, Dr Rudra Dinesh Kumar \\ Dept of Clinical Pharmacy, Chalmeda Anandrao Institute of Institute of Medical Sciences \& Vaageswari \\ College of Pharmacy, Telangana, India \\ *Corresponding Author \\ Dr K. Mahitha Reddy, Pharm.D
}

\begin{abstract}
High blood pressure is ranked as the $3^{\text {rd }}$ most important risk factors for attributable burden of disease in South Asia. Hypertension is responsible for 57\% of stroke deaths in India. Cardiovascular disease will be the largest cause of death and disability in India by 2020. This study aim to analyze the quality of life and medication adherence for hypertensive patients attended tertiary care hospital.

Methods: We conducted prospective observational study on hypertensive patients by use Quality of life and medication adherence questionnaires (minichal Brazil and hill bone complains scale).

Results: The study included 230 populations. The average age group was 65 years old. Out of 230 patients only 30\% of patients came for follow-up and their QOL and medication adherence was compared. The quality of life and medication adherence was increase when compared with previous and $P$ value $\{<0.0001\}$.

Conclusion: The correlation between quality of life and medication adherence was inverse and statistically significant highlighting that greater adherence implies better quality of life. The involvement of clinical pharmacist in providing patient education regarding his condition and medication and life style modification improves patient medication adherence \& quality of life.
\end{abstract}

\section{Introduction}

Hypertension is one of the most preceding problems all over the world, which is contributing to several other health complications like Cardio vascular diseases, Renal Disorders and many other complications. Although Hypertension is a preventable disease, its prevalence has been increasingly rising. Hypertension ranks the first among the preventable causes of death worldwide $^{[1]}$. Awareness about Hypertension and treatment and its control are relatively low worldwide, there are significant differences among different nations ${ }^{[2]}$. Globally hypertension affects approximately one in four adults and results in over ten million deaths annually ${ }^{[3]}$ According to data from the GBD study of 2016, hypertension led to 1.63 million deaths in India in the year 2016 alone. GBD data also showed that over half of the deaths due to ischaemic heart disease $(54.2 \%)$, stroke $(56.2 \%)$ and chronic kidney disease $(54.5 \%)$ were attributable to high systolic $\mathrm{BP}^{[4,5]}$

High blood pressure is ranked as the third most important risk factors for attributable burden of 
disease in South Asia. Hypertension is responsible for 57\% of stroke deaths in India. Cardiovascular disease will be the largest cause of death and disability in India by 2020. Hypertension is emerging as a major health problem ${ }^{[6,7]}$

- North India: The prevalence of hypertension for the rural and urban North Indian population was $14.5 \%$ and $28.8 \%$ respectively.

- East India: The prevalence of hypertension for the rural and urban East Indian population was $31.7 \%$ \& $34.5 \%$ respectively.

- West India: The prevalence of hypertension for the rural and urban West Indian population was $18.1 \%$ \& $35.5 \%$ respectively.

- South India: The prevalence of hypertension for the rural and urban South Indian population was $21.1 \%^{[6,7]}$

Medication adherence is defined as the extent to which a patient's medication taking behavior coincides with the intention of health advice she or he has been given. Medication adherence is one of the most important factors that determine therapeutic out come in patients with chronic illness whatever the efficacy of a drug, it cannot act unless the patient takes it.

Adherence to treatment is the key link between treatment and outcome ${ }^{[9]}$. Patient that were adherent to full regimen of their hypertension treatment were often significantly less likely to have elevated blood pressure ${ }^{[8]}$. Adherence to medications is associated with improved health benefits and patient outcomes.

Non-adherence is the main obstacle for controlling hypertension and a significant barrier to effective hypertension management ${ }^{[10,11]}$.

Several studies conducted globally on Nonadherence have produced a wide range of results. The non-adherence rate in a global study conducted was $45 \%$ and a significant number of the hypertensive patients with co-morbidities were non-adherent to treatment ${ }^{[12]}$. Poor adherence with treatment is one of the main reasons of insufficient response to blood pressure (BP) lowering treatment in patients diagnosed with 'resistant hypertension' (up to $10 \%-20 \%$ of patients with high $\mathrm{BP}^{[13]}$.

Hypertension complications like Renal disorders are mainly due to Non-adherence to the hypertensive meditaions and also maintaining single class of hypertensive drugs without altering the doses for years together.

\section{Materials \& Methodology}

Objectives: The main objective of our study is to evaluate the medication knowledge of the hypertensive patients, their adherence to drug therapy and to assess quality of life.

Study Site: The present study was conducted at OP \& IP of General medicine dept, Chalmeda Ananda Rao institute of medical science, karimnagar, Telangana.

Study Design: Prospective Observational Study

Study Period: 6 months.

Study Criteria: All patients who have diagnosed with hypertension

Inclusion Criteria: All patients with hypertension irrespective of age, gender.

Exclusion Criteria: Critically ill patients who were not in a position to be interviewed, Pregnant woman, Psychiatric patients were excluded from the study.

Scales Used: Minichal Brazilscale, hill bone hbp compliance scales were used in this study. The study was conducted in Chalmeda Ananda Rao institute of medical science for both in-patient (patients on admission) and outpatients of General medicine department. The data includes demographic details and comorbidities and medication using by the patient for hypertension and since how many years were obtained by direct patient interview.

The Quality of life and Medication adherence of each patient was evaluated by using MinichalBrazil scale to know the quality of life this questionnaire has 17 items and two domains (Somatic Manifestations and Mental Status). The answers are distributed in a frequency Liker scale with four response options, from 0 (absolutely 
not) to 3 (yes, very much). On this scale, the closer to 0 is the result, the better the quality of life 1 to 9 questions are included in mental domain score ranges from 0 to 27 score near to 0 indicates good quality of life and score near to 27 indicates worst quality of life, 10 to 16 questions are included in somatic domain the score ranges from 0 to 21 score near to 0 indicates good quality of life and score near to 21 indicates worst quality of life, 17 question indicates overall effect of hypertension and HILLBONE BLOOD PRESSURE COMPLANCE SCALE to no patient medication adherence it consisted of 14 questions/items having 3 subscales under behavioral domains of hypertension treatment which include-Medication adherence, Reduced salt intake and Appointment keeping.

Each question/item was answered with a fourpoint Liker scale ranging from 1 to 4 (4= none of time, 3 =some of the time, $2=$ most of time, and 1 $=$ all the time) and the score ranges from $<25$ (low adherence), 25-35 (moderate adherence) and $>35$ (high adherence).

In these study based on their quality of life was accessed each patient was counselled for 5 to 10 min in verbal form in their native language Telugu. In these counseling we have explained him what is hypertension, causes, and what are the complication associated with it when he stop are use or irregular use of medication. A little changes in Lifestyle can be useful to control blood pressure it includes - Reduce salt intake, Stick to the prescription take your medication regularly, Avoid stress, Do regular exercise, Reducing your alcohol intake, smoking cessesation, Monitoring your blood pressure regularly. By counselling adherence was improved and quality of life was also improved. Life style Modifiaction Information Leaflets were provided to the patient after the counseling.

\section{Statistical Analysis}

- Data analysis was carried out using Microsoft excel 2010 version.

- Statistics was applied using graph pad prism 8.0.2 (263). The correlation between medication adherence and quality of life analyzed using Pearson correlation coefficient (r). The comparison of pre and post counselling medication adherence and quality of life was analyzed using ANOVA.

\section{Results \& Discussion}

A total of 230 patients diagnosed with hypertension and who used some hypertensive treatment were interviewed. In which 151 were male and 79 were females.

Table.no.1: Gender Distribution

\begin{tabular}{|l|c|}
\hline Gender & Population \\
\hline Males & 151 \\
\hline Females & 79 \\
\hline
\end{tabular}

Fig.no.1 Gender Distribution

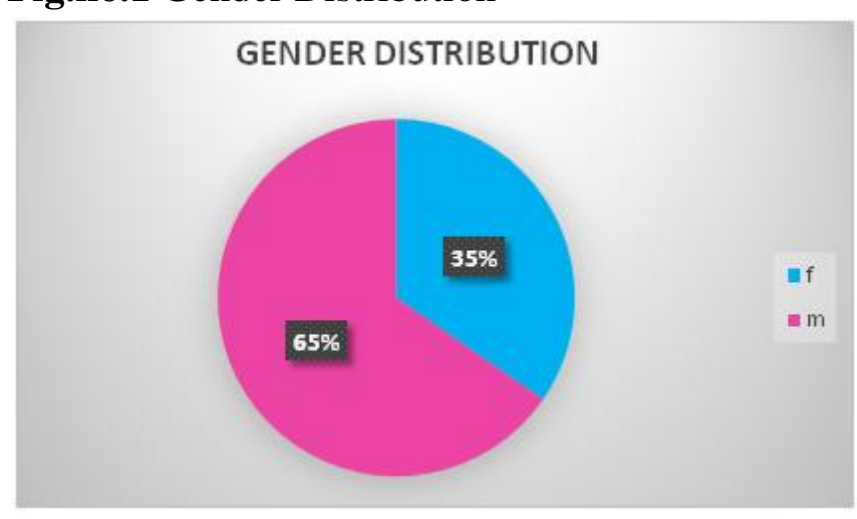

In our study, Age group of 15-30 (0\%), 31$45(12 \%)$, 46-60(36\%), 61-75(43\%), 76-90(9\%) were found.

Table.no.2: Age Distribution

\begin{tabular}{|l|c|}
\hline Age & Patients \\
\hline $15-30$ & $0 \%$ \\
\hline $31-45$ & $12 \%$ \\
\hline $46-60$ & $36 \%$ \\
\hline $61-75$ & $43 \%$ \\
\hline $76-90$ & $9 \%$ \\
\hline
\end{tabular}


Fig. no. 2: Age Wise Distribution

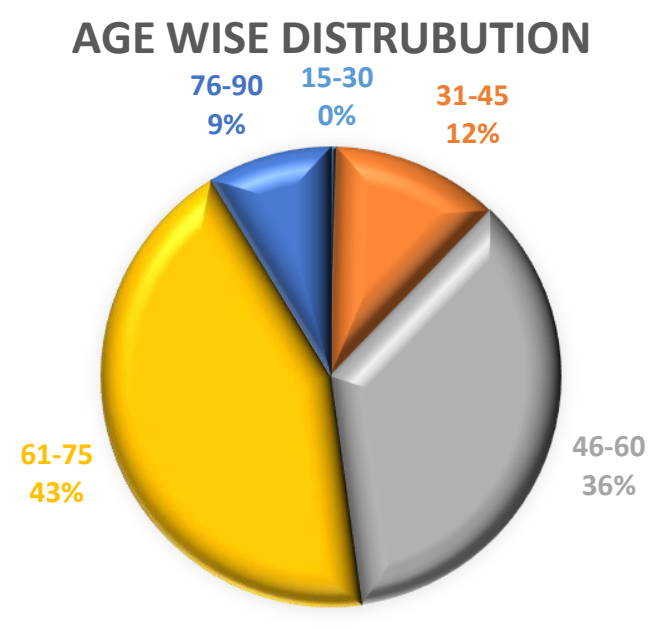

In our study we found that patients of age 60 above are more prone to hypertension than other age groups.

\section{Educational Status of Our Total Population}

Indian is a developing country, literacy rate is slowly progressing. In our study illiterates are $82 \%$. So they are not aware of their medication. More clinical pharmacist attention is required to attain medication adherence
Table no.3: Educational Status

\begin{tabular}{|l|c|}
\hline Educational Status & No.of Subjects \\
\hline Illiterates & 188 \\
\hline Literates & 37 \\
\hline Graduates & 5 \\
\hline
\end{tabular}

Figure.no.3: Educational Status

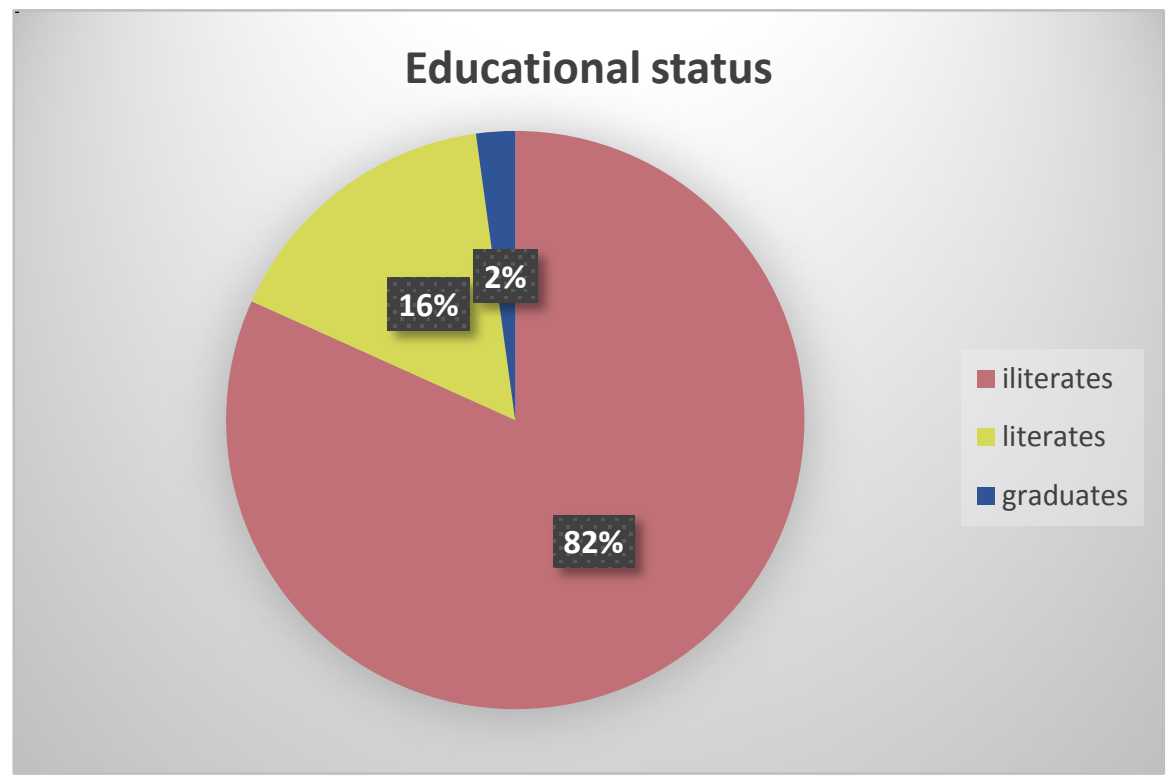

\section{Prescription Patterns of Drugs}

During the study period, 230 prescriptions for hypertension were analyzed and we observed that $95 \%$ of patients are using monotherapy and $5 \%$ are using combination of antihypertensive drugs. Among monotherapy (ARB'S) were prescribed most. Utilization of other major drug classes as mono therapy in decreasing order is alpha blockers (0.8), angiotensin converting enzyme inhibitors (ACE'S) (5.2), beta blockers (13.9), calcium channel blockers(CCB) (29.5). 
Figure.no.4:- Prescription Patterns of Drugs

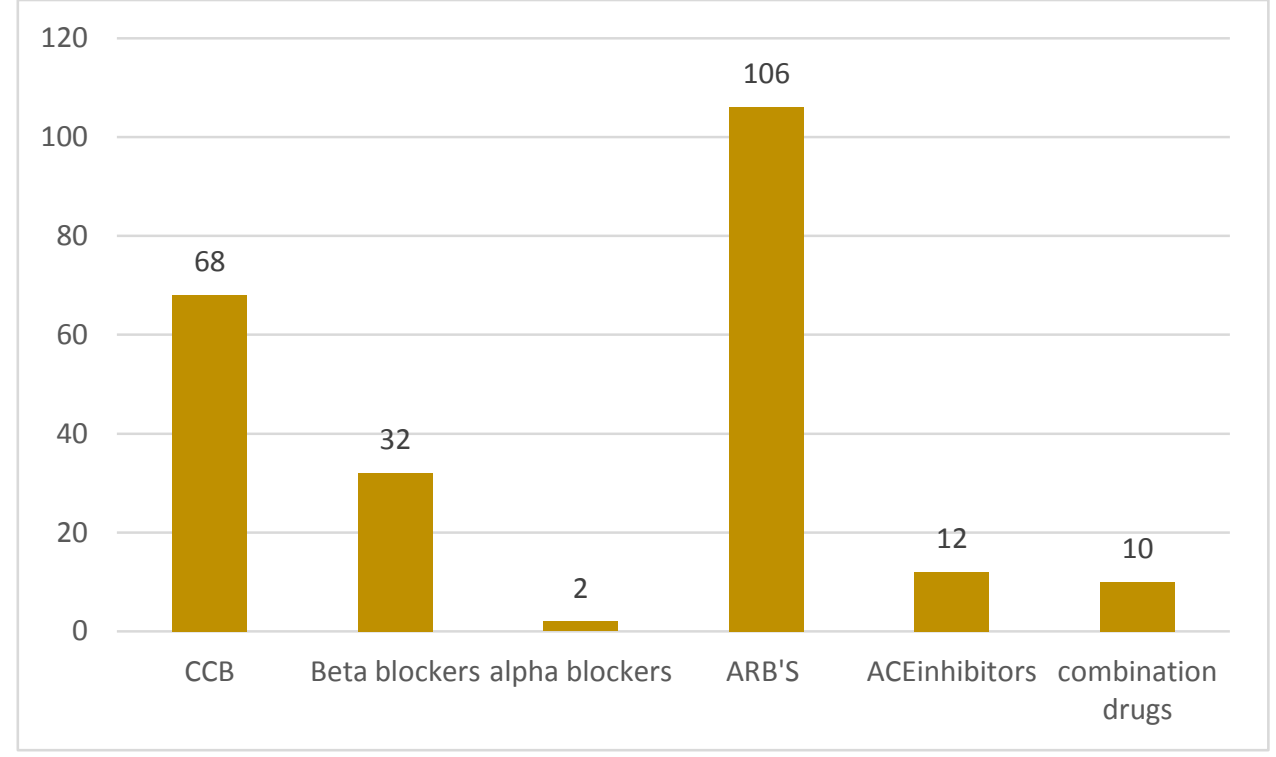

Figure.no.5: Quality of Life in All Patients

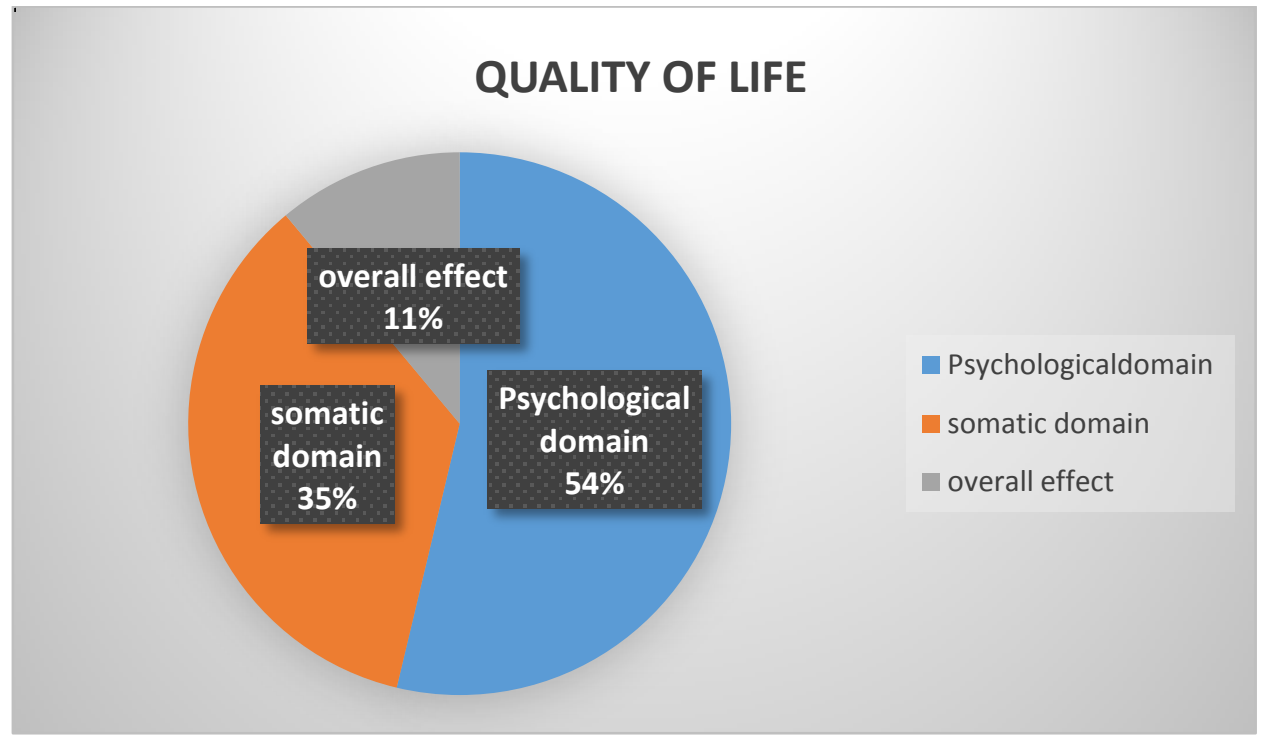

In 230 subjects we observed that most of their quality of life is deteriorated by psychological domain (54\%) which includes decreased sleep, maintenance of social relationships, Decision making, stress \& tension, daily activities, powerlessness. Whereas somatic domain (35\%) include feeling sick, breathlessness for no reason, ankles swelling, frequent urination, dry mouth, pain In chest, tingling sensation and overall effect (11\%) includes HTN effecting QOL. 
Figure.no.6:- Quality of Life in All Patients

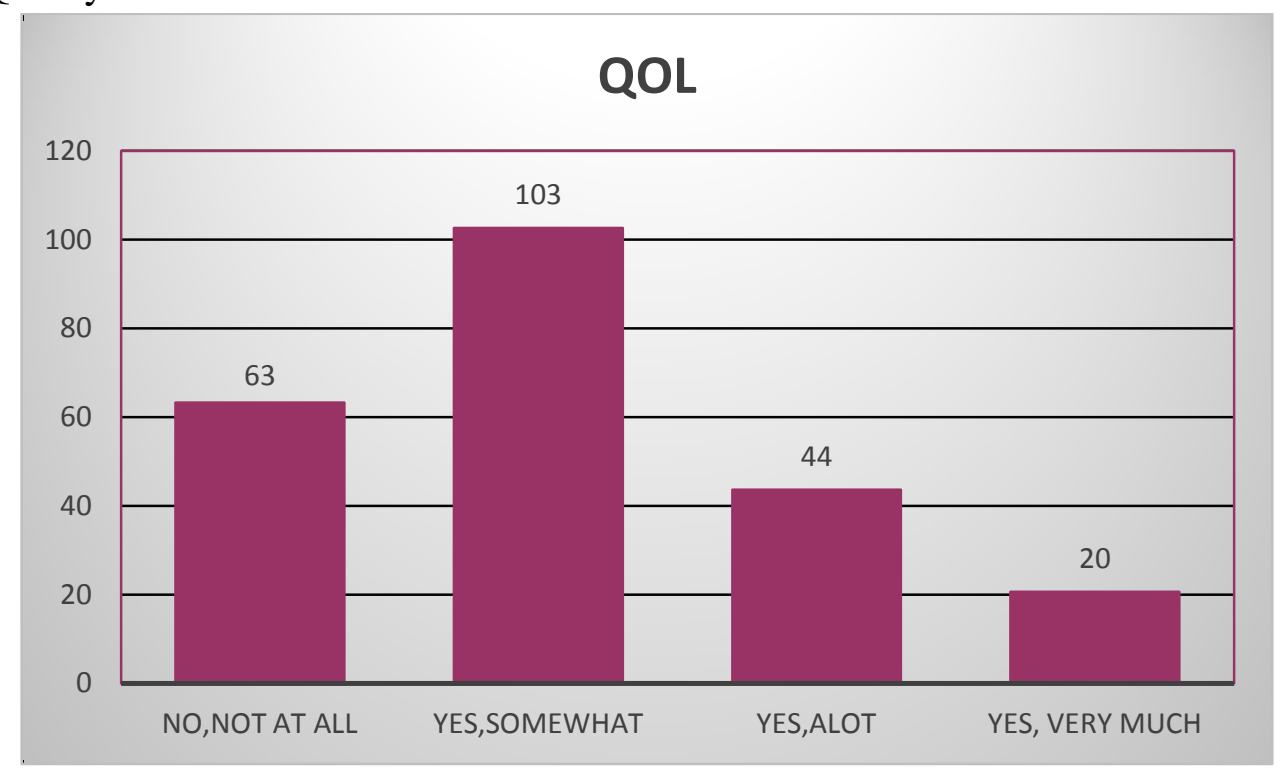

This graph represents the response of patients to all the 17 questions Among 230 subjects 103 subjects said yes somewhat, 63 subjects said no,

Figure.no.7: Medication Adherence In All Patients

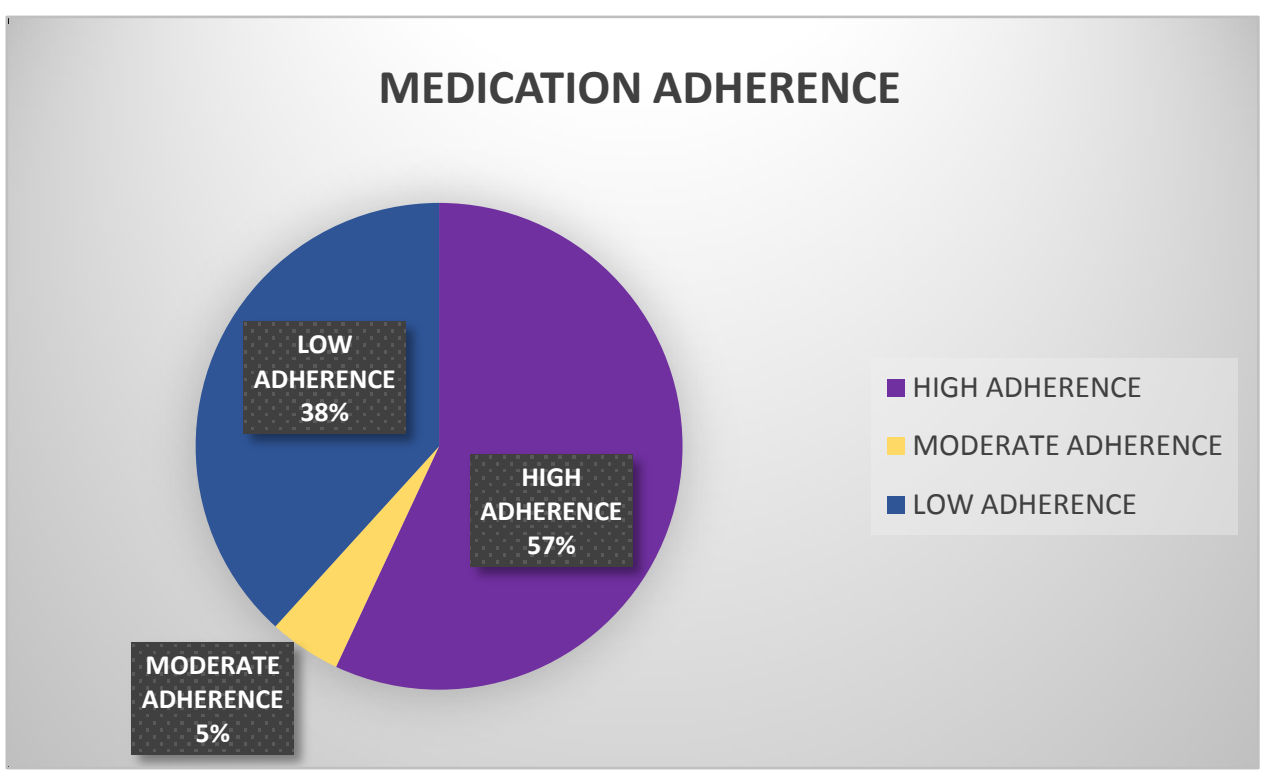

In our observation on total subjects (230) we found that $57 \%$ are high adherent, $38 \%$ are low adherent and $5 \%$ are moderate adherent.

\section{Reviewed Study Population}

Out of 230 cases, 69 patients have attended for second phase so only 69 patients' quality of life and adherence are compared as following: not at all, 44 subjects said yes a lot and 20 subjects replied yes, very much.

\section{0}


Figure.no.8:- Adherence Level before Patient Counselling

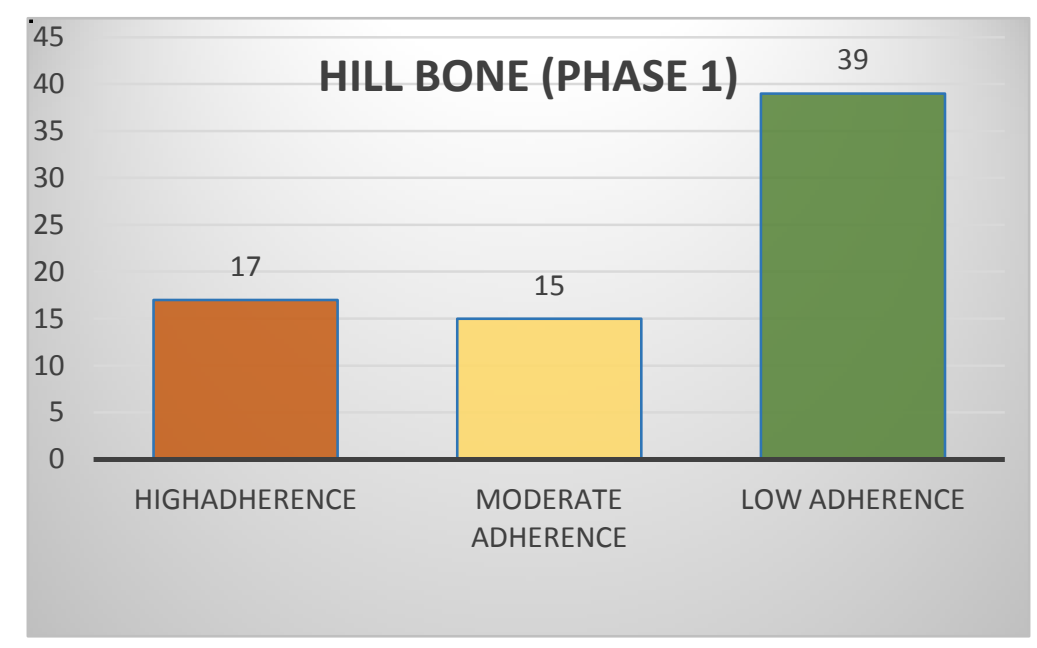

In phase 2: 13, 12, 56 are low, moderate, high respectively

As we can see here, before the counselling patients medication adherence level was low, but after the counselling patient's medication adherences was increased.

Figure.no.9:- Adherence Level after Patient Counselling

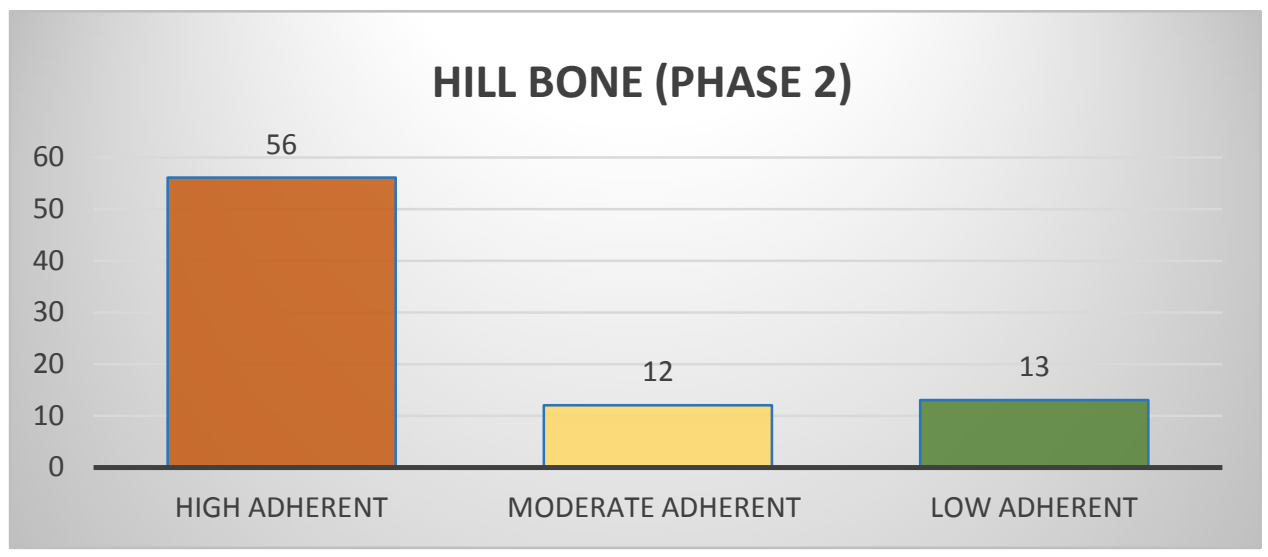

For over all patients came for review their quality of life was as followed

Figure.no.10: Quality of Life Before \& After Patient Counselling

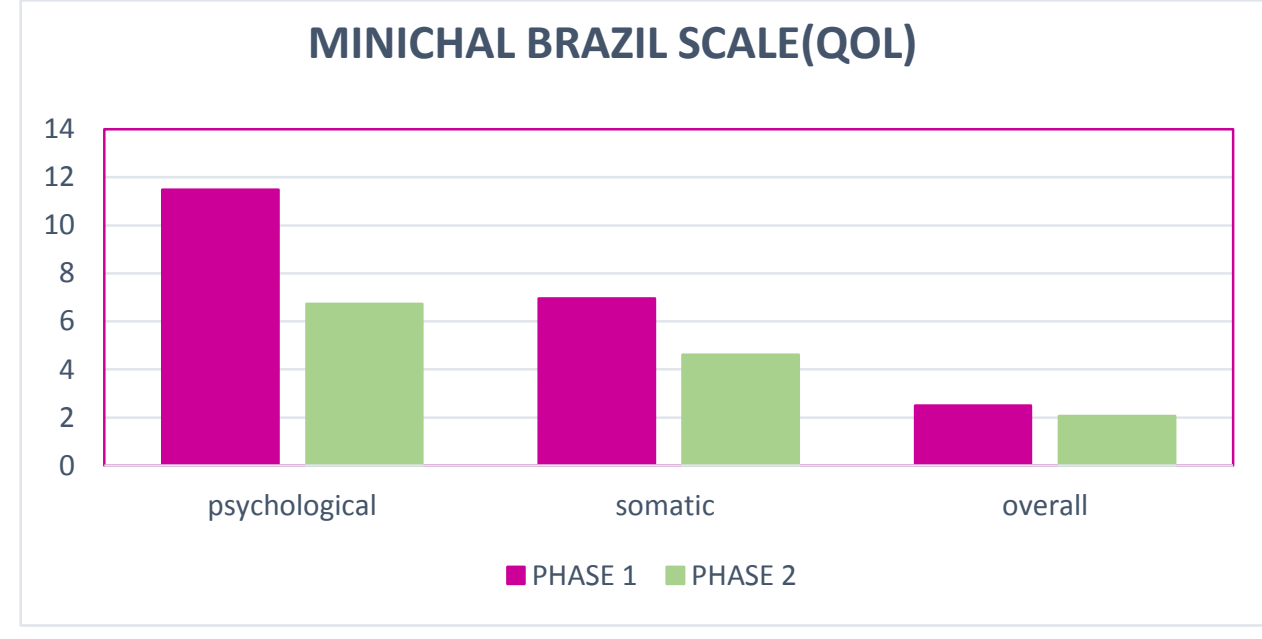


In phase 1 (before counselling) we found that patients have are more disturbance in psychological domain than somatic and overall effect, but in phase 2 (after counselling) patients are again review and we observed that psychological and somatic disturbances were slightly decreased in both psychological domain and somatic domain.

Hypertension is chronic elevation of blood pressure that in the long term causes end organ damage and results in increased morbidity and mortality. Hypertension patients require treatment and life style modification which helps in maintenance of normal blood pressure. Poor adherence leads to decreased quality of life of patient, medication adherence associated with several other parameters is an important factor in achieving blood pressure control.

The Present study was done to assess the quality of life and medication adherence in hypertensive patients to know their medication adherence and quality of life in 230 subjects. Ana Paula et, $\mathbf{a l}^{[14]}$ conducted study on quality of life and medication adherence in hypertensive patients in 13 basic health units on 720 people. Alhaddad IA et, al. ${ }^{[15]}$ conducted study on Treatment adherence and quality of life in patients on antihypertensive medications in a Middle Eastern population: adherence on 1,470 eligible hypertensive patients , Juliét Silveira Hanusv et, al. conducted study on Association between quality of life and medication adherence in hypertensive individuals on 432 hypertensive subjects, Anup Bhusal, et, $\mathbf{a l}^{[16]}$. Conducted study on Assessment of medication adherence among hypertensive patients: a cross-sectional study on 129 subjects medication adherence was poor in hypertensive patients.

In our study we observed $65 \%$ males population were suffering from HTN than females, average age group 61- 75 are mostly seen. In Anapaula et al., study he observed females are more prone to hypertension than males and above 60 age group are mostly seen .in Alhaddad IA et, al. he observed males are more prone to HTN than females In Juliét Silveira Hanusv et al., study he observed females are more with HTN than males, In Anup Bhusal, et al., he observed females are more prone to HTN than males.

As many studies $(14,15,16)$ states that more than $60 \%$ of patients, who have been diagnosed with HTN were on HTN medication; In our study we came to know that $97.3 \%$ patients were on HTN medication.

Combination therapy provides greater protection to target organs than mono therapy. So combination therapy have less side effects $(30)$ so that QOL may increase but in our study population, $82 \%$ were on mono therapy and their QOL is less as stated in fig.no.16. In Alhaddad IA et al., and Anup Bhusal, et al., study they observed most of the patients are on combination therapy than monotherapy.

\section{Acknowledgement}

We would like to thank our Senior Dr. C.Nithish who is the main pillar behind this Work.

\section{Conclusion}

Medication adherence \& quality of life play a major in management of hypertension. The correlation between quality of life and medication adherence was inverse and statistically significant highlighting that greater adherence implies better quality of life. By the involvement of clinical pharmacist in providing patient education towards medication and state of disease, can improve medication adherence and overall quality of life. Through our patient counselling, we have improved the medication knowledge about drugs which the patient is using and made adhere towards it.

\section{References}

1. Yurdakul S, Aytekin S. Hypertension in women. Arch Turkish Soc Cardiol. 2010;38(1):25-31.

2. Abaci A. The current status of cardiovascular risk factors in 
Turkey. Arch Turkish Soc Cardiol. 2011; 39(4):1-5.

3. Dorairaj Prabhakaran, et, al. Prevalence and Incidence of Hypertension: Results from a representative cohort of over 16,000 adults in three cities of South Asia. Indian Heart Journal. July - August 2017, Volume 69 ; (4):434-41.

4. GBD Compare IHME Viz Hub. Global; both series, all ages, 2017, DALYS. Available from: https://vizhub.healthdata.org/gbdcompare/\#, accessed on February 27, 2019.

5. Gupta R, Gaur K, S Ram CV. Emerging trends in hypertension epidemiology in India. $J \quad H u m \quad H y p e r t e n s$ 2018; doi:10.1038/s41371-018-0117-3.

6. Raghupathy Anchala, et.al. Hypertension in India: A systemic review and metaanalysis of prevalence, awareness, and control of hypertension. Journal of Hypertension. 2014 June 32(6), 117077.

7. S.Srinivas et.al. Prevalence of prehypertension in adult population of rural Andhra Pradesh. Asian Journal of Biomedical and Pharmaceutical Science. 2013, vol.3 (23);45.

8. Oza BB, et, al. Health related quality of life in hypertensive patients in a tertiary care teaching hospital. 2014..

9. G Parthasarathi et, al. A Textbook of Clinical Pharmacy Practice, essential concepts and skills. Ch. 6,55-63.

10. Burnier M, Wuerzner G, StruijkerBoudier H, Urquhart J. Measuring, analyzing, and managing drug adherence in resistant hypertension. Hypertension. 2013;62:218-25.
11. van Veen WA. Treatment adherence in hypertension: problems and research. J R Coll Gen Pract Occas Pap. 1980;(12):225.

12. Tadesse M, Eyob A, Akshay S, Asim A. Nonadherence to antihypertensive drugs. A systematic review and meta-analysis. Medicine. 2017;96:e5647.

13. Tomaszewski, M., et al., High rates of nonadherence to antihypertensive treatment revealed by high-performance liquid chromatography-tandem mass spectrometry (HP LC-MS/MS) urine analysis. Heart, 2014: p. heartjnl-2013305063.

14. Ana Paula Ferreira Maciel, et, al. Quality of life and Medication Adherence in Hypertension Patients. October 2016, Vol. 29(5).

15. Alhaddad IA et, al. Treatment adherence and quality of life in patients on Antihypertensive medications in a Middle Eastern, Dove medical press, 27 October 2016 Volume 2016:12 Pages 407-413.

16. Anup Bhusal, et, al. Assessment of medication adherence among Hypertensive Patients: a cross sectional study. International Journal of Basic and Clinical Pharmacology. July-August 2016, Vol. 5(4); 1606-1612. 